$30 \mathrm{~min}$, the quenching efficiency in photofading increases in same order as occurs with quenching in the presence of chemically generated singlet oxygen $(1 e<1 \mathrm{a}<1 \mathrm{f})$. This order may be slightly affected by further irradiation; however, compound $1 \mathrm{f}$ is usually the most effective quencher in photofading.

\section{REFERENCES}

1. GN Schrauzer, Acc. Chem. Res., 2 (1969) 72

2. GN Schrauzer and V P Mayweg, J. Amer. Chem. Soc., 87 (1965) 1483
A Zweig and W A Henderson, J. Polymer Sci., Polymer Chem. Edn, 13 (1975) 717

4. N Kuramoto and T Kitao, J.S.D.C., 98 (1982) 334.

5. J Griffiths and C Hawkins, d.C.S.Perkin II, (1977) 747

6. TDK Corpn, Japan Kokai Tokkyo Koho 84-83695

7. Mitsubishi Electric Corpn, Japan Kokai Tokkyo Koho 80-39340.

8. G N Schrauzer and V P Mayweg, J. Amer. Chem. Soc., 87 (1965) 3585

9. M Kajitani et al., Nippon Kagaku Kaishi (1985) 433.

10. A Davison et al., Inorg. Chem., 3 (1964) 814

11. D Sartain and M R Truter, J. Chem. Soc. (A), (1967) 1264

12. B M Monroe and J J Mrowca, J. Phys. Chem., 83 (1979) 591

13. C S Foote and S Wexler, J. Amer. Chem. Soc. 86 (1964) 3879.

14. N Kuramoto and T Kitao, J.S.D.C., 98 (1982) 159

\title{
Dynamic flow phenomena in a foam application unit
}

\section{A B J Kroezen and J Groot Wassink}

\author{
Twente University of Technology, Enschede, The Netherlands
}

\begin{abstract}
If, during the operation of a foam application unit, a change in the foam production is introduced, the change in the flow becomes noticeable at the point of application only after a certain response delay time. During the operation of the unit it is necessary to take this response delay into consideration. By varying the flow of foam in the application unit, the mass content of foam in the unit is changed as a result of the changed pressure inside the unit. The response delay has been mathematically derived for the flow of a given type of foam through pipes, as well as through a foam printing unit as a whole. A deviation in the yield of foam on the substrate can be avoided by considering the response delay caused by a change in the foam production process.
\end{abstract}

\section{INTRODUCTION}

Dynamic flow phenomena such as response delay are aspects of the operation of foam application units calling for special attention during start-up, shut-down or a change in the production process. After a certain delay time, a change in the foam production in the mixer of an application unit affects the quantity of foam leaving the unit at the point of application. This delay in response is caused by a change in the mass content of foam in the application unit, which in turn is a function of pressure drop, itself related to change of flow. The subsequent interactions are due to a combination of the compressibility and the relatively high viscosity of the foam.

Response delay can lead to deviations in the yield of foam on the substrate. In many application units the yield is determined directly from the quantity of foam flowing to the point of application (particularly in closed application systems). Response delay becomes less important in those systems where a foam buffer is present at the application point.

An example of a process in which response delay becomes problematic is foam printing. In this process the foamed paste passes through a distribution slit and is applied on the substrate using a rotary screen [1,2]. The printing process is started up at a low production speed. After checking the primary printing result, adjustment to a higher production speed is made. In order to meet the required yield specifications during the phase of adjustment, the response delay that may appear must be considered and accounted for. When the foam production and fabric speed are increased simultaneously, they can only be increased slowly in order to keep the yield on substrate within the limits set by the specifications [2]. However, if the response delay time is taken into account by firstly increasing the foam production and secondly the fabric speed, it is possible to increase the fabric speed and still satisfy the yield requirements.

The way in which response delay affects certain physical quantities in foam application units has been derived mathematically, based on a description of the flow profile of the foam in application units [3]. The derivation applies to the dynamic flow of a particular foam system in pipes as well as to flow in the entire unit. The dynamic flow can be described by a partial differential equation, which can be solved numerically. In this way a simulation program is obtained by which the dynamic flow can be calculated. Based on step functions of the flow entering the unit, the response delay curves of the flow leaving the unit have been determined as a function of several parameters. The different response curves resulting from the step functions can each be standardised to a response curve to give the dimensionless flow number as a function of the dimensionless time number. Finally a typical problem in process control is solved as an example of how calculations of the dynamic flow may be used to solve practical problems in foam printing.

\section{STATIONARY FLOW OF FOAM}

Foam is a viscous and compressible colloidal dispersion with particular rheological properties. The flow of foam in 
the laminar flow regime through a pipe, or through an entire application unit, may be calculated from a knowledge of the rheological properties of the foam. The actual flow rate of foam and the blow ratio in the foam are dependent on the local pressure. The rheology of the foam varies as a function of the actual blow ratio, and may thus be used as a function of the latter [3].

The rheological values used in the derivation were obtained from viscosity measurements on a foamed solution which was composed of $10 \mathrm{~g} / \mathrm{l}$ lauryl sulphate and thickened with $30 \mathrm{~g} / 1$ Solvitose FN (a starch ether). The rheology of the foam can be described by the well known power law $\left(\tau=k D r^{n}\right)$. The experimentally determined values of the rheological constants $k$ and $n$ (as a function of the blow ratio) are given in Figure 1. (The power law may also be written as $\tau=k_{2}\left(D_{\mathrm{r}} / D_{\mathrm{r}, 0}\right)^{n}$, where $D_{\mathrm{r}, \mathrm{o}}$ is the reference shear rate. In Figure 1 the $k_{2}$ value given is for $D_{\mathrm{r}, 0}=800 \mathrm{~s}^{-1}$.)

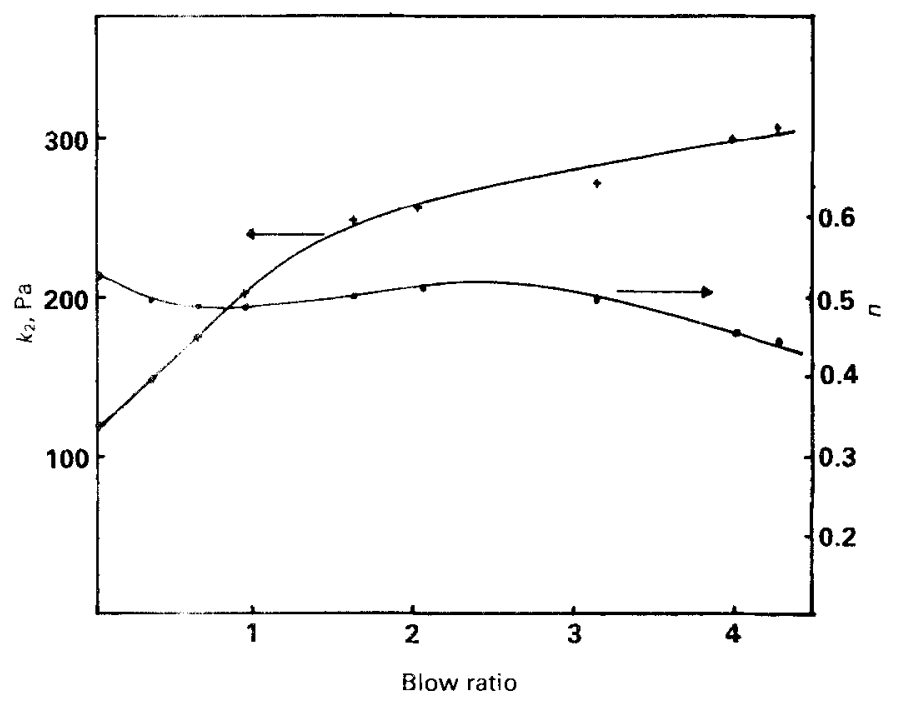

Figure 1 -- Rheological properties of the foam used

The influence of changes in the bubble diameter on the rheological properties was ignored, and calculations were carried out for a foam with a mean bubble diameter of about $40 \mathrm{~km}$. Slip effects at the wall are of minor importance in this system because of the small ratio of foam viscosity versus liquid viscosity [3].

Based on the equation of flow for a pseudoplastic, the pressure drop over a pipe can be determined numerically. With a pressure drop of several atmospheres over the pipe the actual blow ratio and the volumetric flow rate change considerably over the length of the pipe. Eqn 1 is therefore integrated numerically over the pipe length:

$$
\begin{gathered}
\frac{\mathrm{d} P}{\mathrm{dX}}=-\left(F_{\mathrm{v}} \frac{3 n+1}{\pi n} R^{-(3 n+1) / n}\right)^{n} 2 k \\
F_{\mathrm{v}}=F\left(1+B_{\circ} \frac{P_{0}}{\mathrm{P}}\right) / \rho_{1}
\end{gathered}
$$

Furthermore, the rheological constants are a function of the actual blow ratio $\left[B_{\circ}\left(P_{\circ} / P\right)\right]($ Eqn 3$)$ :

$$
n, k=\mathrm{f}(B)
$$

The initial conditions are given in Eqns 4 and 5:

$$
\begin{gathered}
x=1 \quad P=1 \mathrm{~atm} \\
F(\mathrm{x})=\text { constant }
\end{gathered}
$$

As for the pipe, the pressure drop over the entire unit can be determined in a similar way. The foam printing machine is made up of three sections: the mixer, the transport tube and the distribution section. The mixer has a certain volume but a negligible pressure drop. The transport pipe, on the other hand, usually has a considerable pressure drop of several atmospheres. The distribution section consists of two parallel pipes both having a narrow slit (Figure 2). The slit diameter changes over the length of the pipe resulting in an even distribution over the entire width of the application unit. In the foam printing machine represented, the space between the slit and the point of application is rather small and therefore the correspondingly small pressure drop is neglected in the calculations.

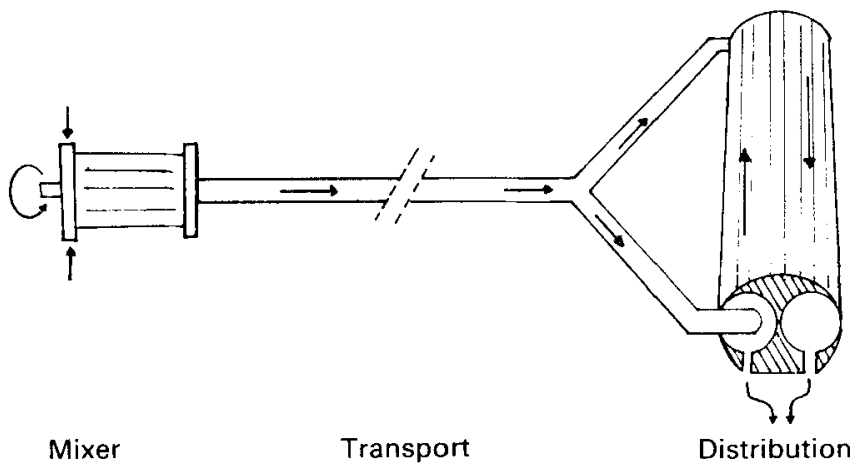

Figure 2 - Simple layout of a foam printing machine

In order to describe the flow through the narrow slit use is made of an empirical relationship (Table 1). The consequences on the microscopic level of the flow in narrow slits has been evaluated in an earlier publication [3]. The data as used in the calculations are given in Table 1.

\section{TABLE 1}

\section{Data used in calculations ${ }^{(a)}$}

\begin{tabular}{lc}
\hline \multicolumn{1}{c}{ Parameter } & Value \\
\hline Foam density $(\mathrm{g} / \mathrm{l})$ & 200 \\
Blow ratio & 4 \\
& \\
Dimensions of foam printer & $0.6 \times 10^{-3}$ \\
Mixer volume $\left(\mathrm{m}^{3}\right)$ & 8 \\
Transport pipe length $(\mathrm{m})$ & 0.017 \\
Transport pipe diameter $(\mathrm{m})$ & 1.6 \\
Feed pipe length $(\mathrm{m})$ & 0.017 \\
Feed pipe diameter $(\mathrm{m})$ & 1.6 \\
Distribution pipe length $(\mathrm{m})$ & 0.025 \\
Distribution pipe diameter $(\mathrm{m})$ & $0.3-0.6$ \\
Slit diameter variation $(\mathrm{mm})$ &
\end{tabular}

(a) Equation for flow in slit:

$$
\Delta P=a(F / b)^{c} . D^{d}
$$

where

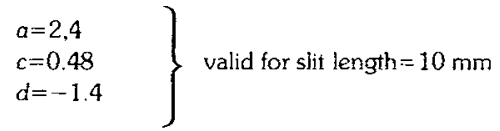

\section{DYNAMIC FLOW OF FOAM}

During constant flow through a pipe the pressure drop reaches one stable value. By suddenly changing the flow rate a pressure wave arises which moves slowly through the pipe. After a certain time the pressure drop over the pipe adjusts itself to a new stable value, corresponding to 
the new flow rate. When calculations based on these phenomena are made, the results are described in terms of the variable $x$, designating the location in the pipe, and the variable $t$, giving the time. The pressure drop in the pipe and the corresponding flow rate are thus solutions to a partial differential equation.

In deriving the differential equation the mass balance over a segment $i$ of the pipe with length $\Delta x$ follows from the fact that accumulation equals flow in minus flow out (Eqn 7):

$$
\frac{\delta\left(v_{i} \rho_{\mathrm{s}, i}\right)}{\delta t}=F_{\mathrm{in}}-F_{\text {out }}
$$

Prior to any further derivation Eqn 7 can be written in the form of Eqn 8:

$$
A \frac{\delta \rho_{\mathrm{s}}}{\delta t}=\frac{\delta F}{\delta x}
$$

where $\delta v_{i}=A \delta x$

As was the case for constant flow, Eqns 2 and 3 are valid. The system can therefore be described by the following set of equations, comprising a partial differential equation, additional equations, and initial and boundary conditions.

\section{Differential equation}

$$
\frac{B_{\mathrm{o}} P_{\mathrm{o}}}{\left(P+B_{\mathrm{o}} P_{\mathrm{o}}\right)^{2}} \cdot \frac{\delta P}{\delta t}=-\frac{\delta}{A \delta x}\left(\frac{c\left(\frac{\delta p}{\delta x}\right)^{1 / n}}{\left(1+B_{\mathrm{o}} P_{\mathrm{o}} / P_{\mathrm{o}}\right)}\right)
$$

Additional equation

$$
\begin{gathered}
c=\frac{n}{3 n+1} \pi R^{-(3 n+1) / n} \cdot\left(\frac{1}{2 k}\right)^{1 / n} \\
n=\mathrm{f}(B) \\
k=\mathrm{f}(B)
\end{gathered}
$$

Initial and boundary conditions

$$
\begin{array}{ll}
t=0 & P(x)=\text { stationary flow } \\
x=1 & P(l)=1 \text { atm } \\
x=0 & F(t)=\text { step function }
\end{array}
$$

The equations were solved numerically using the well known Euler method. Calculations are made in steps with magnitudes $\Delta x$ and $\Delta t$. The choice of $\Delta x$ and $\Delta t$ determines on one hand the size of the segmentational error, and on the other hand the numerical stability of the calculation system. The $\Delta x$ and $\Delta t$ values must be chosen in such a manner that residence time of the foam per segment is much longer than the time step $\Delta t$, in order to avoid numerical instability. Subsequently the convergence of the calculating system may be examined by varying $\Delta x$ and $\Delta t$. In the examples used a value of $20 \mathrm{~cm}$ was chosen for $\Delta x$, and $\Delta t$ was chosen in the interval 0.001 to $0.01 \mathrm{~s}$. For this interval the calculations proceed- ed without any problems. The dynamic flow may also be derived in a similar way for the entire foam printing unit.

Quite apart from the term describing the viscous resistance, Eqn 9 should include a term related to inertia. Estimations made in the examples show, however, that inertia does not contribute much to flow resistance. This is mainly caused by the relatively low density compared with the high viscosity of the foam.

\section{PRESSURE WAVES AND RESPONSE CURVES}

Calculations have been made for the flow through a pipe of $8 \mathrm{~m}$ long and a diameter of $0.02 \mathrm{~m}$. The mass flow rate was increased from $F_{\min }$ to $F_{\max }$. The step function introduces a pressure wave, which travels slowly through the pipe (Figure 3). The flow rate of foam leaving the pipe can be represented by a response curve (Figure 4). When the wave reaches the final segment of the pipe the flow leaving the pipe starts to increase. Subsequently the flow increases to the new stationary value. The results of the calculations are given in the Figures 3-7 and in Table 2.

\section{TABLE 2}

\section{Calculations on response curves}

\begin{tabular}{cllcc}
$\begin{array}{c}\text { Calculation } \\
\text { No. }\end{array}$ & $\begin{array}{c}F_{\min } \\
(\mathrm{kg} / \mathrm{s})\end{array}$ & $\begin{array}{c}F_{\max } \\
(\mathrm{kg} / \mathrm{s})\end{array}$ & $F_{\max } / F_{\min }$ & $\begin{array}{c}\text { Response time } \\
(\boldsymbol{\Phi}=1)(\mathrm{s})\end{array}$ \\
\hline 1 & 0.01 & 0.05 & 5 & 3.70 \\
2 & 0.01 & 0.1 & 10 & 2.54 \\
3 & 0.01 & 0.02 & 2 & 5.68 \\
4 & 0.05 & 0.1 & 2 & 1.62 \\
5 & 0.001 & 0.002 & 2 & 27.6 \\
6 & 0.05 & 0.04 & 0.8 & 2.38 \\
7 & 0.01 & 0.03 & 3 & 4,75 \\
\hline
\end{tabular}

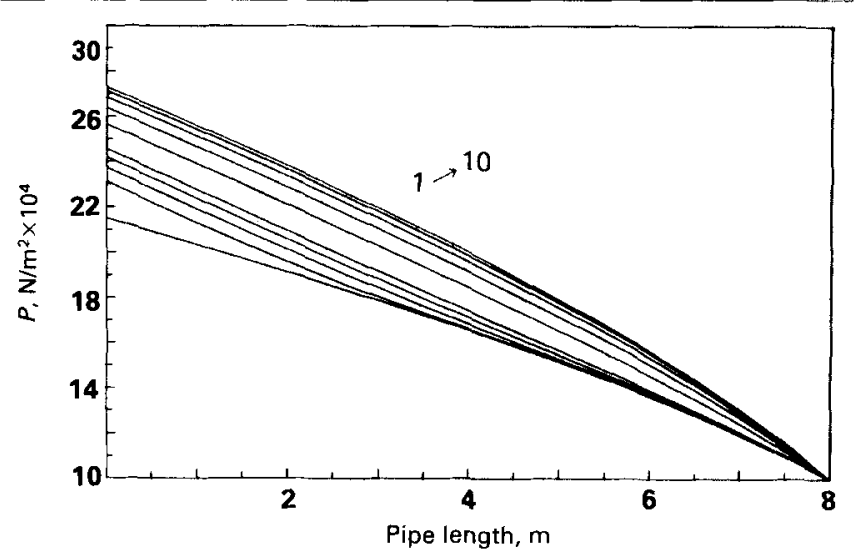

\begin{tabular}{lcccccccccc} 
Curve & 1 & 2 & 3 & 4 & 5 & 6 & 7 & 8 & 9 & 10 \\
\hline$\theta$ & 0 & 0.08 & 0.16 & 0.24 & 0.32 & 0.64 & 0.96 & 1.28 & 1.6 & 1.92
\end{tabular}

Figure 3 - Pressure wave in the pipe (calculation 7)

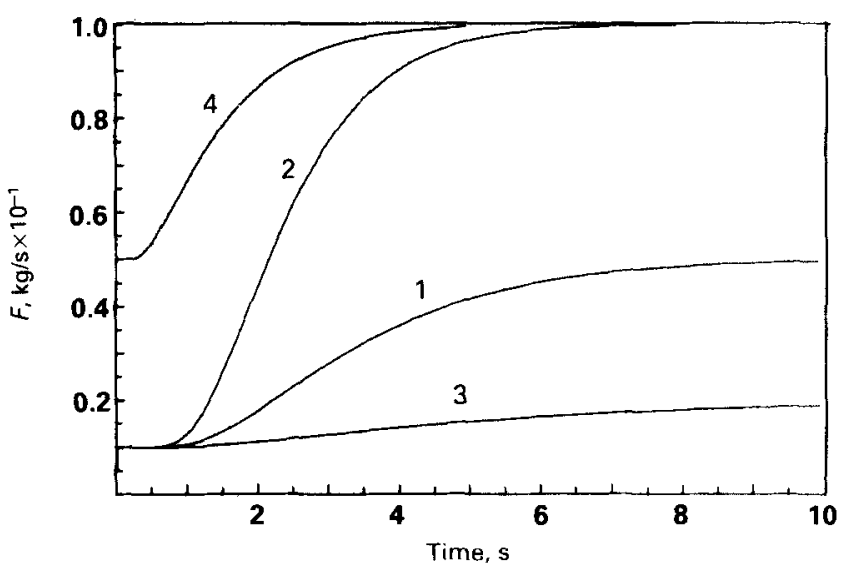

Figure 4-Response curves (calculations 1-4) 
STANDARDISATION OF THE RESPONSE CURVES

In order to continue the analysis, the response curves resulting from an increase in flow from $F_{\min }$ to $F_{\max }$ were standardised to response curves, where the dimensionless flow number $\phi$ is given as a function of the dimensionless time number $\theta$. The delay in response is a result of an increase of the mass of foam contained in the pipe (mass content). One relationship that is important for the standardisation gives the mass content of the pipe as a function of flow rate at constant flow conditions (Eqn 16):

$$
\text { Mass }=\mathrm{f}(F)
$$

The difference between the incoming flow and the outgoing flow, integrated over time, is equal to the increase of mass content of the pipe (Eqn 17):

$$
\operatorname{Mass}\left(F_{\text {max }}\right)-\operatorname{mass}\left(F_{\text {min }}\right)=\int_{0}^{\infty}\left(F_{\text {in }}-F_{\text {out }}\right) \mathrm{d} t
$$

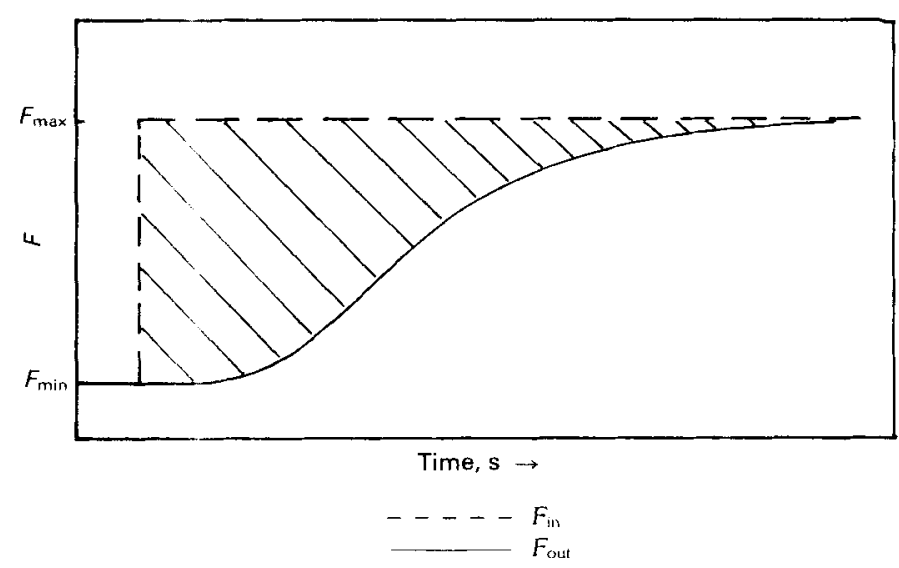

Figure 5 - Increase of mass content in transient response

This is represented by the shaded area in Figure 5 .

With Eqn 13 the two dimensionless numbers specified in Eqns 18 and 19 may be defined:

$$
\begin{gathered}
\phi=\left(F_{\text {out }}-F_{\text {min }}\right) /\left(F_{\text {max }}-F_{\text {min }}\right) \\
\theta=t\left(F_{\text {max }}-F_{\text {min }}\right) /\left[\text { mass }\left(F_{\text {max }}\right)-\text { mass }\left(F_{\text {min }}\right)\right]
\end{gathered}
$$

Figures 6 and 7 show the standardised response curves using the values of $F_{\min }$ and $F_{\max }$ as given in Table 2. The corresponding response time, when $\theta=1$ are also given in Table 2 . These response times can be calculated directly from Eqn 16. Figures 6 and 7 show that the shape of the response curve is nearly independent of the magnitude of the flow. However, the shape is strongly influenced by the ratio $F_{\max } / F_{\text {unin }}$. A larger value of $F_{\max } / F_{\min }$ indicates a steeper breakthrough curve.

It is also clear that the response time increases with decreasing flow rate or increasing period of delay of the foam in the unit.

\section{ADJUSTMENT SCHEME FOR THE FOAM PRINT. ING UNIT}

Dynamic flow phenomena, as they appear in the whole foam printing unit, are analogous to those for flow through pipes. Thus the response curves resulting from a change in foam production can be calculated in a similar way. These response curves appear to be of about the same shape as those resulting from transient flow through pipes and therefore will not be treated any further in this article.

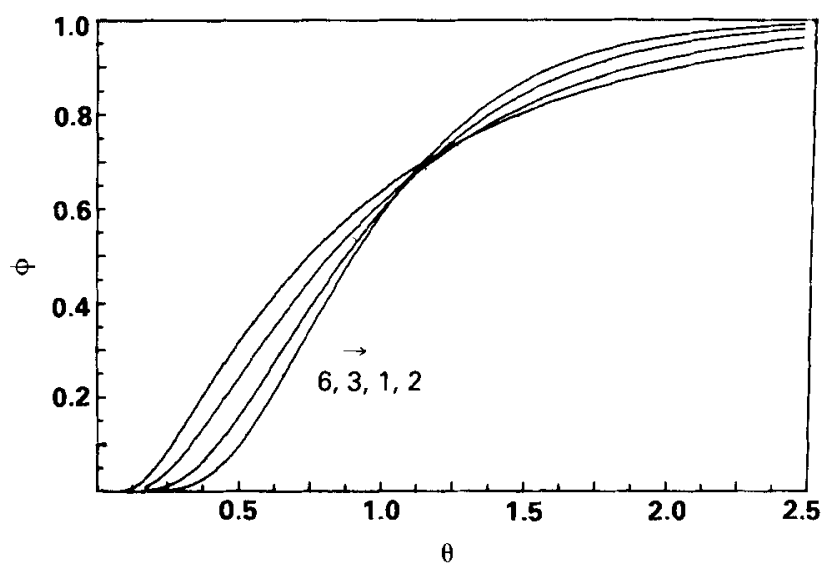

Figure 6 - Standardised response curves (calculations 1-3, 6); $\mathrm{F}_{\max } / \mathrm{F}_{\min }=10,5,2$ and 0.8

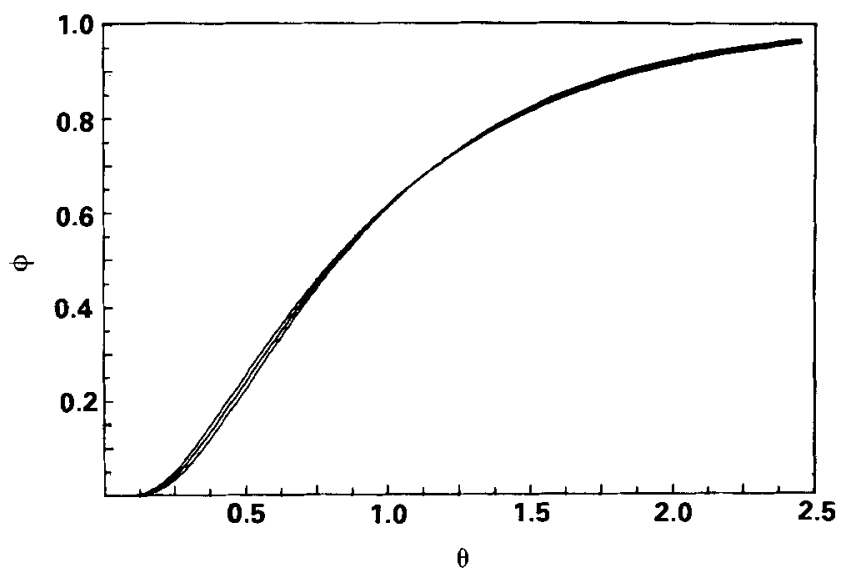

Figure 7 - Standardised response curves (calculations 3-5) $\mathrm{F}_{\max } / \mathrm{F}_{\min }=$ constant

Instead we will examine a practical problem in foam printing. It will be shown how foam production can be controlled in such a way that the apparent response delay is compensated for. In the foam printing example, the machine is started with a fabric velocity of $4 \mathrm{~m} / \mathrm{min}$ while $0.5 \times 10^{-3} \mathrm{~kg} / \mathrm{s}$ foam throughout is applied. After checking the primary printing results the velocity of the fabric is linearly increased to $40 \mathrm{~m} / \mathrm{min}$ in $45 \mathrm{~s}$, during which time the yield on the substrate remains the same.

A procedure, based on the knowledge of the response delay and the shape of the response curves resulting from a step function, may be used to obtain an increased production speed, the foam production being increased by increments. The magnitude of the increments, as well as the time intervals at which they are applied, can be determined as follows.

(a) The mixer production is increased to a higher rate of flow by means of a step function. The step function is applied at the moment at which the time interval from the preceding step function equals the breakthrough time from the preceding step $(\theta=1)$.

(b) The magnitude of the step $F_{i}$ to $F_{i+1}$ is chosen so that the desired yield after the breakthrough time equals $0.5 \times\left(F_{i}+F_{i+1}\right)$.

(c) The magnitude of the first step function $\left(F_{0}\right.$ to $\left.F_{1}\right)$ is chosen so that the response curve follows the 
desired yield curve up to $\theta=1$. This will not be the same for the second step, so the second step function $\left(F_{1}\right.$ to $\left.F_{2}\right)$ is different.

The result of the calculations of this example is given in Figure 8, which indicates that the damping action of the foam averages out during each individual step as was expected. The resulting function closely resembles the desired ramp function.

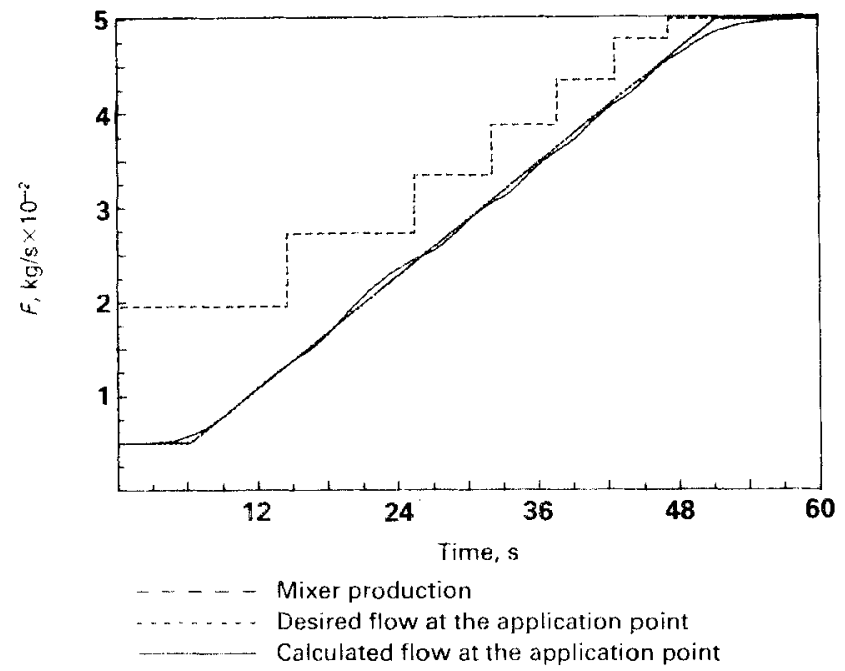

Figure 8 - Example of a production scheme

For the first two steps in the given procedure only the equation which relates mass to flow is used. In the third step of the procedure use is also made of the shape of the response curves (Figures 6 and 7). The scaling-up scheme is built up of iterative calculated steps. Uneven distribution of foam over the width of the substrate may be caused by the pressure wave in the distribution unit. In the example chosen this effect is not very great. At much higher accelerations, however, this may become a problem.

In order to be able to set up a scaling-up scheme for practical purposes, the rheology of the foam should be known. However, a simpler method is to determine Eqn 16 empirically. This can be carried out by measuring the pressure drop in the foam printing unit as a function of the flow rate. When the pressure in every part of the machine and the machine delay are known, the mass and the response delay times can be calculated easily.

It becomes apparent that a printing unit having a relatively large internal volume, and at rather small flow rates, will lead to a large response delay time. For this reason, in order to optimise the controllability, the printing unit should be designed so that the delay period of the unit, and thus the foam content, should be kept to a minimum. A system that controls the response delay should be integrated in the printing machine. The machine must contain specially designed start-up, shutdown and scaling-up programs in order to compensate for dynamic flow phenomena. Some commercial foam application machines have already been provided with such programs. Within a given range of viscosities of foam, various types of start-up, shut-down and scaling-up programs may be used, which are stored in the control system.

\section{REFERENCES}

1. C J Blaak, Melliand Textilber, 11 (1985) 809.

2. B Jones, J D Tumer and L. G Snijder, Textile Industries (Oct 1984) 25.

3. A B J Kroezen and J Groot Wassink, J.S.D.C., Accepted for publication.

\section{LIST OF SYMBOLS}

Symbol
$A$
$a, c, d$
$B, B_{\circ}$

Description

Unit

Cross-sectional area of the flow

$\mathrm{m}^{22}$

Constants in slit flow equation

Actual blow ratio and blow ratio at

atmospheric pressure (volumetric ratio gas/liquid)

D Diameter of slit of distribution unit $\mathrm{m}$

$D_{\mathrm{r}} \quad$ Shear rate $\mathrm{s}^{-1}$

$F \quad$ Mass flow rate $\mathrm{kg} / \mathrm{s}$

$\mathrm{F} / \mathrm{b} \quad$ Flow rate per meter width $\mathrm{kg} / \mathrm{ms}$

$F_{\text {in }}, F_{\text {out }}$ Incoming and outgoing mass flow $\mathrm{kg} / \mathrm{s}$

$F_{\min }, F_{\max }$ Mass flow prior to and right after the step function supplied

$F_{\mathrm{v}} \quad$ Volume flow of foam

\section{$\mathrm{kg} / \mathrm{s}$}

$F_{i}, F_{i+1} \quad$ Mass flow after ith or $(i+1)$ st step in production scheme

$m^{3 / s}$

$k \quad$ Rheological value

$\mathrm{kg} / \mathrm{s}$

Pas"

$k_{2} \quad$ Shear stress at reference shear rate

$$
\left(D_{\mathrm{r}}=800 \mathrm{~s}^{-1}\right)
$$

$\mathrm{Fa}$

$L \quad$ Length of pipe

III

Rheological content

Pressure

$P$

$P_{\mathrm{o}}$

$R$

Atmospheric pressure

\section{$N / m^{2}$}

$\mathrm{N} / \mathrm{m}^{2}$

Inside radius of a tube

$m$

Time

Volume

Coordinate of length of the pipe

$s$

$\mathrm{m}^{3}$

$\mathrm{m}$

$\begin{array}{ll}x & \text { Coordinate of length of the } \\ \phi & \text { Dimensionless flow number }\end{array}$

$\rho_{\mathrm{s}}, \rho_{1} \quad$ Density of foam and liquid respectively $\mathrm{kg} / \mathrm{m}$

$\theta \quad$ Dimensionless time number

$\tau$ Shear stress $\mathrm{N} / \mathrm{m}^{2}$ 